\title{
Inclusion of a time-dependent, non-local convection theory in a stellar evolution code
}

\author{
Achim Weiss ${ }^{1}$ and Martin Flaskamp ${ }^{1}$ \\ ${ }^{1}$ Max-Planck-Institut für Astrophysik, Karl-Schwarzschild-Str. 1, 85748 Garching, Germany \\ email: aweiss@mpa-garching.mpg.de
}

\begin{abstract}
The non-local, time-dependent convection theory of Kuhfuß (1986) in both its oneand three-equation form has been implemented in the Garching stellar evolution code. We present details of the implementation and the difficulties encountered. Specific test cases have been calculated, among them a $5 M_{\odot}$ star and the Sun. These cases point out deficits of the theory. In particular, the assumption of an isotropic velocity field leads to too extensive overshooting and has to be modified at convective boundaries. Some encouraging aspects are indicated as well.
\end{abstract}

Keywords. Convection, methods: numerical, stars: evolution

\section{The Kuhfuß theory}

R. Kuhfuß (Kuhfuß 1986, 1987a,b) developed a one-dimensional model for describing stellar convection with the aim of presenting a non-local and time-dependent theory for use in stellar evolution programs. The theory is based on expanding the fluctuations in the hydrodynamics equation in terms of moments, stopping at the second order by closure conditions for the third order moments. The theory results in three partial differential equations for the mean kinetic energy $\omega=\left\langle\frac{1}{2} \vec{v}^{2}\right\rangle$, the mean entropy excess $\Phi=\left\langle\frac{1}{2} s^{\prime 2}\right\rangle$, and the correlation between velocity and entropy fluctuations $\vec{\Pi}=\left\langle\vec{v}^{\prime} s^{\prime}\right\rangle$. Including the closure conditions, one arrives at (see Kuhfuß 1986 and Flaskamp 2003 for details):

$$
\begin{gathered}
d_{\mathrm{t}} \omega=\frac{\nabla_{\mathrm{ad}} T}{H_{p}} \Pi-\frac{C_{D}}{\Lambda} \omega^{\frac{3}{2}}+\frac{1}{\rho} \operatorname{div} j_{\mathrm{t}} \\
d_{\mathrm{t}} \Pi=\frac{2 \nabla_{\mathrm{ad}} T}{H_{p}} \Phi+\frac{2 c_{p}}{3 H_{p}}\left(\nabla-\nabla_{\mathrm{ad}}\right) \omega-\frac{\beta_{\Pi}}{\Lambda} \omega^{\frac{1}{2}} \Pi-\frac{1}{\tau_{\mathrm{rad}}} P i \\
d_{\mathrm{t}} \Phi=\frac{c_{p}}{H_{p}}\left(\nabla-\nabla_{\mathrm{ad}}\right) \Pi-\frac{\beta_{\phi}}{\Lambda} \omega^{\frac{1}{2}} \Phi-\frac{2}{\tau_{\mathrm{rad}}} \Phi
\end{gathered}
$$

These are the three equations, Flaskamp (2003) is solving within the Garching stellar evolution code to compute the convective flux and the convective gradient in full stellar models. They are integrated into the standard implicit Henyey-scheme, which implies a coupling of three grid points instead of two. The additional numerical variables are, due to the nature of the physical variables (they can become negative), arcsinh-transformations of the physical quantities. In addition, $\omega$ is transformed to $\sqrt{\omega}$. Chemical mixing is done with a diffusion scheme using the convective velocity of the theory. As in previous investigations we find that the new system of equations is numerically much less stable, and convergence problems abound.

Kuhfuß (1986) showed that by assuming the connection $\vec{\Pi} \approx-\alpha_{s} \Lambda \sqrt{\omega} \nabla\langle s\rangle$ one can reduce the system of three equations to a one-equation theory: 

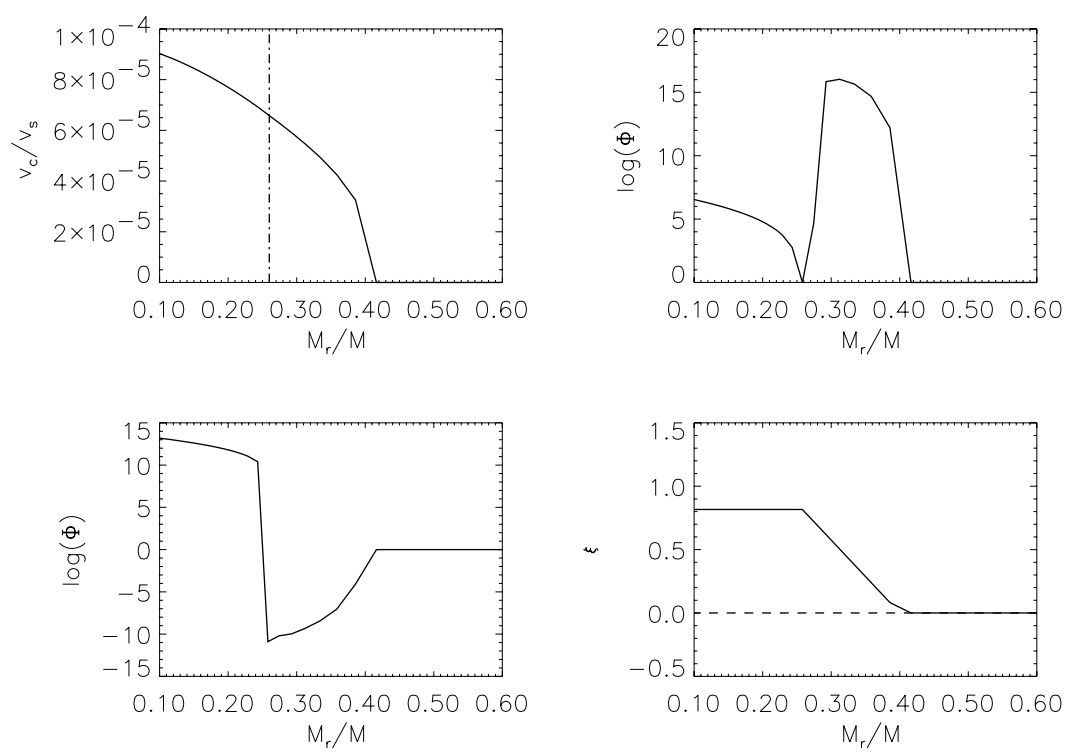

Figure 1. Convective core structure of a $5 M_{\odot}$ ZAMS star. The run of the isotropy parameter $\xi$ (lower right) has been imposed on the three-equation theory. The vertical line in the upper left panel would be the classical Schwarzschild boundary.

$$
d_{\mathrm{t}} \omega=\frac{\nabla_{\mathrm{ad}} c_{p} T \Lambda \alpha_{s}}{H_{p}^{2}} \omega^{\frac{1}{2}}\left(\nabla-\nabla_{\mathrm{ad}}\right)-\frac{C_{D}}{\Lambda} \omega^{\frac{3}{2}}+\frac{1}{\rho} \operatorname{divj} \mathrm{j}_{\mathrm{t}}
$$

In this form radiative losses are no longer included, but can be added (Wuchterl 1995). The parameters $C_{D}, \beta_{\Pi}, \beta_{\phi}$ of the theory are now correlated by the additional parameter $\alpha_{s}$. The convective flux $j_{t}$ contains a further parameter $\left(\alpha_{t}\right)$, which turned out to be critical. Kuhfuß estimated a value of 0.25 for it.

\section{Applications}

Applying the three equations theory straightforwardly to a ZAMS star of $5 M_{\odot}$ results in a convective core that encompasses the whole star. An analysis of the details revealed that the assumption of an isotropic velocity field is responsible for this unrealistic behaviour. In fact, the correlation between radial velocity and kinetic energy directly determines the extent of overshooting (Fig. 1).

Wuchterl (1995) and Wuchterl \& Feuchtinger (1998) have introduced further improvements and extensions to the theory, which were also used here: (a) radiative losses by connecting radiative diffusion with convective energy via the radiative diffusion timescale; (b) limitation of the mixing length scale to the distance to the nearest convective boundary, as otherwise it would diverge at the stellar center; (c) a flux limiter, since the theory allows enthalpy transport being faster than the convective velocity; this transport speed was therefore limited by Wuchterl (1995).

To test the time-dependency of the theory, we followed the onset of convection in a $5 M_{\odot}$ star at the beginning of He-burning, using, due to numerical difficulties, the one-equation version only. We could nicely follow the onset of convection (Fig. 2, left). However, the almost dynamical core helium flash in a low-mass star could even in this case 

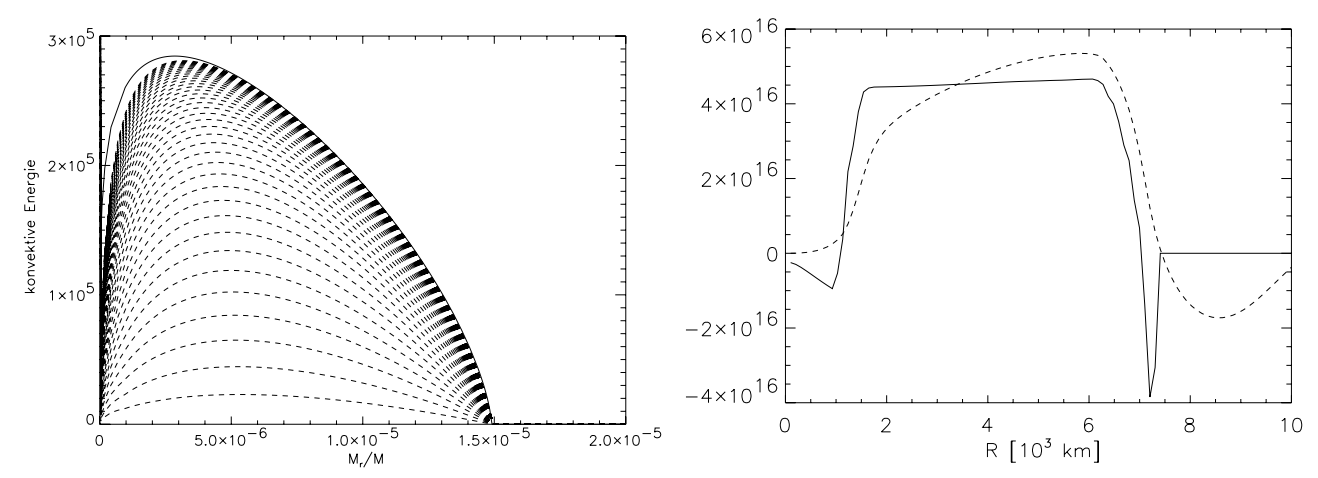

Figure 2. Left: Onset of helium core convection in a $5 M_{\odot}$ star. Shown is the convective energy in steps of 22 days (time steps between the dashed lines) and the asymptotic stationary solution (solid). Right: Convective flux in an A-star envelope of $T_{\text {eff }}=8000 \mathrm{~K}$ in our own model (solid) and a hydro-simulation by Muthsam (1999; dashed).

be followed only until the helium luminosity reached $\log L_{\mathrm{He}}>8$. The flash developed about $20 \%$ faster and more violent due to the non-instantaneous convective transport.

The final example we show concerns the overshooting in an A-star envelope (oneequation theory). In Fig. 2 (right) we compare the convective flux (solid line) with that resulting from 3-d hydro simulations by Muthsam (1999; dashed). While a certain similarity is evident, the details, in particular in the overshooting layers differ appreciably. Both approaches predict that the two convective layers are connected due to overshooting. As Kupka \& Montgomery (2002) we find a non-vanishing convective velocity but negligible convective flux between the two convective regions of an $8000 \mathrm{~K}$ hot A-star.

\section{Summary}

(i) The three equation version of the Kuhfuß-theory is a numerical challenge, if implemented into a full stellar evolution code. (ii) Various tests, including an intermediate-mass ZAMS star, reveal that the resulting overshooting is much too large and additional restrictions/assumptions to the theory are needed. (iii) The one-equation version is simpler, easier to implement, and results in more realistic non-local effects. (iv) Comparison with hydro-simulations and Kupka \& Montgomery (2002) shows an encouraging agreement for the structure of A-type star envelope convection. (v) However, a solar model test by Flaskamp (2003) is passed only if one of the parameters of the theory, $\alpha_{t}$, is reduced to a value of 0.1. (vi) Although the theory has promising aspects, the need for additional fine-tuning and the numerical difficulties presently prevent a practical advantage over using MLT.

\section{References}

Flaskamp, M. 2003, PhD (Tech. Univ. Munich)

Kuhfuß, R. 1986, A\&A 160, 116

Kuhfuß, R. 1987, PhD (Tech. Univ. Munich)

Kupka, F. \& Montgomery, M. 2002, MNRAS 330, L6

Muthsam, H. 1999, New Astron. 4, 405

Straka, C. 2002, PhD (Univ. Heidelberg)

Wuchterl, G. 1995, Comp. Phys. Comm. 89, 119

Wuchterl, G. \& Feuchtinger, M. 1998 A $\& A$ 340, 419 